\title{
Preface to the Special Issue on Recommender Systems
}

\author{
Tommaso Di Noia ${ }^{1}\left(\mathbb{D} \cdot\right.$ Dietmar Jannach $^{2}$
}

Published online: 9 January 2016

(C) Springer-Verlag Berlin Heidelberg 2016

Recommender systems have become a part of our daily life when interacting, e.g., with Web sites and mobile applications. While originally conceived to suggest new items to buy in e-commerce settings, they are nowadays a fundamental building block of many personalized information access systems. This Special Issue on Recommender Systems of the Journal of Data Semantics includes the extended versions of three selected papers from the Search, Comparison and Recommender Systems track of the 16th International Conference on Electronic Commerce and Web Technologies (EC-Web 2015) which took place in September 2015, in Valencia, Spain. The papers were selected by taking into account the quality, significance and relevance of the results they present. All the extended papers went through an additional peer review process.

The paper "Using Implicit Preference Relations to Improve Recommender Systems" by Ladislav Peska and Peter Vojtas focuses on preference elicitation and learning from implicit feedback. The proposed approach is tailored to scenarios where only a small number of user interactions are available for personalization. The authors show how their model, which is based on partial preference ordering (Implicit Preference Relation), can be used in combination with other recommendation techniques to improve the accuracy of the recommendations. An offline experimental evaluation on two datasets shows the effectiveness of the proposed approach.

Tommaso Di Noia

tommaso.dinoia@poliba.it

Dietmar Jannach

dietmar.jannach@tu-dortmund.de

1 Politecnico di Bari, Bari, Italy

2 TU Dortmund, Dortmund, Germany
In their paper "Product-Seeded and Basket-Seeded Recommendations for Small-Scale Retailers" Marius Kaminskas et al. propose an item-centric personalization approach which is especially relevant for scenarios with a small amount of returning customers. The work represents an industrial case study of applying recommendation functionality to two small Web shops. The approach is evaluated in both an offline and an online A/B test and the results show that the applied association rules technique together with a similarity based approach outperform the pre-configured recommendation strategy of the NitroSell platform.

The third paper (published in Volume 5, Issue 2, pp 99-113), "Content-based Video Recommendation System based on Stylistic Visual Features", by Yashar Deldjoo et al. addresses the problem of video recommendation. The proposed approach is based on the comparison of visual features that are extracted from videos such as Average shot length, Color variance, Motion and Lighting Key. The adoption of these features, borrowed from the field of content-based video retrieval, is quite novel in the recommender systems arena. An interesting result presented in the paper is that extracting and using the features from the trailer of a movie can be sufficient for recommendation purposes. The authors can finally show that the usage of visual features in a simple content-based recommender system can outperform a recommendation approach that uses the genres of a movie as content features.

\section{Guest Editors}

